\title{
Bending Analysis of Micropolar Beams
}

\author{
K.N. Betancourt, Bachelor degree, K. Soncco, Bachelor degree, and R. Arciniega, Ph.D. \\ Universidad Peruana de Ciencias Aplicadas (UPC), Peru, u201410150@upc.edu.pe, u201412519@upc.edu.pe, \\ roman.arciniega@upc.edu.pe
}

\begin{abstract}
The purpose of this research is to study the bending behavior of micropolar beams by using an improved first-order shear deformation theory. The proposed formulation employs five independent variables for the displacement field and a single parameter for microrotations. The model considers thickness stretching and $3 D$ constitutive parameters. A finite element formulation is developed with spectral interpolation functions to avoid shear and Poisson locking. Convergence analysis of vertical deflections is presented to illustrate the performance of high-order elements. Numerical results obtained for cantilever and simply supported beams demonstrate the validity of the present approach.

Keywords-Micropolar elasticity, Micropolar beams, Nonclassical continuum.
\end{abstract}

\section{INTRODUCTION}

Due to the fast development in technology, there is a growing need in the design and manufacturing of micro and nano-electromechanical systems like biosensors, microsensors, or microscopes. Specifically, the use of beamlike elements is general for those systems. This small-scaled structure develops size-dependent behaviors that cannot be evaluated by classical elasticity theories [1], [2]. For this, Shaw [3] has concluded an increasing hardening in microbending plates due to their stiffness parameters. Cantilever nanowires and polymer nanotubes were experimentally tested, resulting in an increase in the elastic modulus when smaller samples were analyzed [4]. Lam et al. [5], [6] and Mcfarland and Colton [6] concluded that the rigidity of microcantilever beams has an inverse dependency on the thickness of such elements. These experimental researches have led to the further development of theories that include intrinsic length scales important to capture nano and microscale phenomena. A general overview of these theories can be found in reference [7].

In reference [8], Voigt included the effects of momentbased stresses in classical elasticity theory. Cosserat and Cosserat [9] developed a new theory considering three new independent rotations, known as microrotations and three displacements in every point of the continuum, leading to a new concept of non-symmetric elasticity theory. The references [10]-[13] further developed micropolar elasticity.

Applications of this theory in beams by using the Finite Element Method can be found in the literature. Nampally et al. [14] developed a non linear micropolar beam solutions using lattice core beams. In reference [15] a linear micropolar model was used for the static bending analysis of a cantilever beam.

Digital Object Identifier (DOI):

http://dx.doi.org/10.18687/LACCEI2020.1.1.472

ISBN: 978-958-52071-4-1 ISSN: 2414-6390
A dynamic analysis considering a Timoshenko beam model was also studied [16]. In reference [17], the micropolar model was extended to torsion as well as bending of cantilever and simply supported beams and results were obtained through the Finite Element Method. Both an static and dynamic analysis considering von Kármán nonlinearity was evaluated analytically [18]. In reference [19], high-order beam models were developed. This theory was also used for the analysis of lattice core sandwich beams considering von Kármán nonlinearity [14] and a geometrically exact model [20], also using the finite element method. From the literature review, it is seen that the bending analysis of micropolar beams has been widely studied. However, there is limited evidence of the use of higher-order models for this kind of studies.

This research aims to present a computational model for the analysis of micropolar beams. The theoretical formulation is based on the improved first-order shear deformation theory (IFSDT) The adopted beam formulation can be found in [21]. The propos herein used considers five independent variables for the approximation of the displacement field and one for the microrotation field. This beam formulation will allow the use of $3 \mathrm{D}$ constitutive equations. A finite element formulation was derived using spectral high-order interpolation functions.

\section{THEORETICAL FORMULATION}

\section{A. Linear isotropic micropolar theory}

Being $\mathbf{v}$ the displacement and $\boldsymbol{\vartheta}$ the microrotation field respectively of a continuum and considering spatial gradients are infinitesimal

$$
\begin{aligned}
& \|\mathrm{v}\| \ll 1, \quad \| \text { Gradv } \| \ll 1 \\
& \|\vartheta\| \ll 1, \quad \| \text { Gradv } \| \ll 1,
\end{aligned}
$$

the linear stretch tensor $\boldsymbol{\varepsilon}$ and the linear wryness tensor $\boldsymbol{\gamma}$, with I as the identity tensor in the undeformed configuration, are defined as follows [22]

$$
\boldsymbol{\varepsilon}=\operatorname{Gradv}-\boldsymbol{\vartheta} \times \mathbf{I}, \quad \gamma=\boldsymbol{G r a d} \boldsymbol{\theta} .
$$

For a linear micropolar isotropic solid, stress-strain relations for the stress tensor $\boldsymbol{\sigma}$ and the couple stress tensor $\chi$ are stated as [23]

$$
\begin{gathered}
\boldsymbol{\sigma}=\lambda \mathbf{I} \operatorname{tr} \boldsymbol{\varepsilon}+(\bar{\mu}+\kappa) \boldsymbol{\varepsilon}+\bar{\mu} \boldsymbol{\varepsilon}^{T}, \\
\chi=\beta_{1} \mathbf{I t r} \boldsymbol{\gamma}+\beta_{2} \boldsymbol{\gamma}^{T}+\beta_{3} \boldsymbol{\gamma},
\end{gathered}
$$


where $\bar{\mu}, \lambda, \kappa, \beta_{1}, \beta_{2}, \beta_{3}$ micropolar elastic constants. Parameters $\bar{\mu}$ and $\kappa$ relates to the Lamé shear modulus $\mu$ according to [24] as

$$
\mu=\bar{\mu}+\frac{1}{2} \kappa
$$

and $\lambda$ is the first Lamé parameter.

Furthermore, Young's modulus $E$ and Poisson's ratio $v$ can be defined in terms of these micropolar elastic constants by the following equations

$$
\begin{gathered}
E=\frac{(2 \bar{\mu}+\kappa)(3 \lambda+2 \bar{\mu}+\kappa)}{2 \lambda+2 \bar{\mu}+\kappa}, \\
\nu=\frac{\lambda}{2 \lambda+2 \bar{\mu}+\kappa} .
\end{gathered}
$$

In (3) $\operatorname{tr}(\cdot)$ represents the trace operation.

\section{B. Beam theory}

Let $\left\{x^{i}\right\}$ be a set of Cartesian coordinates with orthonormal basis $\left\{\mathbf{e}_{i}\right\}$. The $x^{1}$ axis is aligned with the horizontal axis of the beam, passing through the centroid of the cross-section, $x^{3}$ goes through its thickness and $x^{2}$ is transversal to it. The displacement and microrotation fields are assumed to be of the following form (see [21])

$$
\begin{gathered}
\mathbf{v}\left(x^{1}, x^{3}\right)=\mathbf{u}\left(x^{1}\right)+x^{3} \boldsymbol{\varphi}\left(x^{1}\right)+\left(x^{3}\right)^{2} \boldsymbol{\Psi}\left(x^{1}\right), \\
\boldsymbol{\vartheta}\left(x^{\mathbf{1}}\right)=\boldsymbol{\theta}\left(x^{\mathbf{1}}\right) .
\end{gathered}
$$

where $\mathbf{u}=u_{i} \mathbf{e}_{i}$ represents the displacement vector of the neutral axis, while $\boldsymbol{\varphi}=\varphi_{i} \mathbf{e}_{\boldsymbol{i}}$ and $\boldsymbol{\psi}=\psi_{3} \mathbf{e}_{3}$ are difference vectors with $i=1,3$. The displacement field contains five independent variables and contains one quadratic term $\boldsymbol{\psi}$ to avoid poisson locking. The microrotation field contains $\boldsymbol{\theta}=\theta_{3} \mathbf{e}_{3}$, which represent the microrotation along the transversal axis of the beam.

For the given displacement and microrotation fields presented in equations (6) and (7) and by the first equation in (2), the linear stretch tensor is defined as

$$
\begin{gathered}
\varepsilon=\varepsilon^{(0)}+x^{3} \varepsilon^{(1)}, \\
\varepsilon^{(i)}=\varepsilon_{11}^{(i)} \mathbf{e}_{1} \otimes \mathbf{e}_{1}+\varepsilon_{22}^{(i)} \mathbf{e}_{2} \otimes \mathbf{e}_{2} \\
+\varepsilon_{12}^{(i)} \mathbf{e}_{1} \otimes \mathbf{e}_{2}+\varepsilon_{21}^{(i)} \mathbf{e}_{2} \otimes \mathbf{e}_{1},
\end{gathered}
$$

where $i=0,1$, while the linear wryness tensor is expressed by means of the second equation in (2) as

$$
\begin{gathered}
\gamma=\gamma^{(0)}, \\
\gamma^{(0)}=\gamma_{13}^{(0)} \mathbf{e}_{1} \otimes \mathbf{e}_{3},
\end{gathered}
$$

where high order terms are neglected.
It is also possible to express equations (8) and (9) in indicial notation considering the five components of the displacement field and the single component of the microrotation field as

$$
\begin{array}{cc}
\varepsilon_{11}^{(0)}=u_{1,1} & \varepsilon_{11}^{(1)}=\varphi_{1,1} \\
\varepsilon_{22}^{(0)}=\varphi_{2} & \varepsilon_{22}^{(1)}=2 \psi_{2} \\
\varepsilon_{12}^{(0)}=u_{2,1}-\theta_{3} & \varepsilon_{12}^{(1)}=\varphi_{2,1} \\
\varepsilon_{21}^{(0)}=\varphi_{1}+\theta_{3} & \varepsilon_{21}^{(1)}=0 \\
\gamma_{13}^{(0)}=\theta_{3,1} & \gamma_{13}^{(1)}=0
\end{array}
$$

\section{Principle of virtual work}

The weak form of the model is constructed by the Principle of Virtual Work, in which virtual stress measures are work-conjugate to virtual strain measures in the micropolar continuum, as stated in [25]. The configuration solution of the micropolar beam is defined by the set $\Phi \equiv(\mathbf{u}, \boldsymbol{\varphi}, \boldsymbol{\Psi}, \boldsymbol{\theta})$. Thus,

$$
\begin{aligned}
\mathcal{G}(\Phi, \delta \Phi)= & \mathcal{G}_{\text {int }}(\Phi, \delta \Phi)-\mathcal{G}_{\text {ext }}(\Phi, \delta \Phi) \\
\mathcal{G}(\Phi, \delta \Phi)= & \int_{x^{1}}\left(\mathbf{N}^{(0)} \cdot \delta \boldsymbol{\varepsilon}^{(0)}+\mathbf{N}^{(1)} \cdot \delta \boldsymbol{\varepsilon}^{(1)}\right. \\
& \left.+\mathbf{M}^{(0)} \cdot \delta \boldsymbol{\gamma}^{(0)}\right) d x^{1} \\
& +\int_{x^{1}}(\mathbf{p} \cdot \delta \mathbf{u}+\mathbf{m} \cdot \delta \boldsymbol{\theta}) d x^{1}
\end{aligned}
$$

where $\delta \Phi \equiv(\delta \mathbf{u}, \delta \boldsymbol{\varphi}, \delta \boldsymbol{\psi}, \delta \boldsymbol{\theta}) . \mathbf{N}^{(i)}$ are the force stress resultants, $\mathbf{M}^{(0)}$ is the micropolar stress resultant, $\mathbf{p}$ are the body forces acting on the beam per unit length and $\mathbf{m}$ are the body moment forces respectively.

Force stress resultants $\mathbf{N}^{(i)}$ are of the form

$$
\mathbf{N}^{(i)}=\sum_{j=1}^{1} \mathbb{B}^{(i+j)} \boldsymbol{\varepsilon}^{(j)}, \quad i=0,1,
$$

while moment stress resultants $\mathbf{M}^{(0)}$ have the form

$$
\mathbf{M}^{(i)}=\mathbb{M}^{(\mathbf{0})} \gamma^{(\mathbf{0})} \text {. }
$$

For the definition of $\mathbf{N}^{(i)}$ and $\mathbf{M}^{(0)}$ it is necessary to define the material stiffness coefficients $\mathbb{B}$ and $\mathbb{M}$ as

$$
\begin{gathered}
\mathbb{B}^{(k)}=\int_{-h / 2}^{h / 2}\left(x^{3}\right)^{k} \mathbb{C} d x^{3}, \quad k=0,1,2, \\
\mathbb{M}^{(0)}=h \mathbb{D},
\end{gathered}
$$

where $h$ represents the thickness of the beam.

The new fourth order micropolar tensors $\mathbb{C}$ and $\mathbb{D}$ are stated by means of the definitions from [23]

$$
\begin{gathered}
\mathbb{C}=\lambda \mathbf{I} \otimes \boldsymbol{I}+(\bar{\mu}+\kappa) \mathbf{e}_{\boldsymbol{a}} \otimes \mathbf{e}_{b} \otimes \mathbf{e}_{\boldsymbol{a}} \otimes \mathbf{e}_{b} \\
+\bar{\mu} \mathbf{e}_{\boldsymbol{a}} \otimes \boldsymbol{I} \otimes \mathbf{e}_{\boldsymbol{a}}
\end{gathered}
$$




$$
\begin{aligned}
\mathbb{D}=\beta_{1} \boldsymbol{I} \otimes \boldsymbol{I}+\beta_{2} \mathbf{e}_{\boldsymbol{a}} \otimes \boldsymbol{I} \otimes \mathbf{e}_{\boldsymbol{a}} \\
+\beta_{3} \mathbf{e}_{\boldsymbol{a}} \otimes \mathbf{e}_{\boldsymbol{b}} \otimes \mathbf{e}_{\boldsymbol{a}} \otimes \mathbf{e}_{\boldsymbol{b}},
\end{aligned}
$$

where $a, b=1,2,3$.

From equations (11) to (13), the final expression of the virtual work can be stated as

$$
\begin{aligned}
\mathcal{G}(\Phi, \delta \Phi)=\int_{x^{1}} & \sum_{i=0}^{1} \sum_{j=0}^{1} \delta \boldsymbol{\varepsilon}^{(i)} \mathbb{B}^{(i+j)} \boldsymbol{\varepsilon}^{(j)} d x^{1} \\
& +\int_{x^{1}} \delta \boldsymbol{\gamma}^{(0)} \mathbb{M}^{(\mathbf{0})} \boldsymbol{\gamma}^{(\mathbf{0})} d x^{1} \\
& -\int_{x^{1}}(\mathbf{p} \cdot \delta \mathbf{u}+\mathbf{m} \cdot \delta \boldsymbol{\theta}) d x^{1}
\end{aligned}
$$

\section{FINITE ELEMENT APPROXIMATION}

Let $\Omega$ be the domain of the neutral axis of the beam which is discretized into $N$ elements such that

$$
\Omega=\bigcup_{e=1}^{N} \Omega^{\mathrm{e}}
$$

Recall that $\widehat{\Omega}^{e}=[-1,1]$ is a parent domain in $\xi$-space and $x^{1}(\xi): \widehat{\Omega}^{e} \in \mathbb{R} \rightarrow \Omega^{\mathrm{e}}$. The finite element equations are obtained by interpolating the components of the field variables written in terms of the base vectors. Namely,

$$
\begin{aligned}
& \mathbf{u}^{h p}\left(x^{\mathbf{1}}\right)=\left(\sum_{j=\mathbf{1}}^{\boldsymbol{m}} u_{k}^{(j)} \phi^{(j)}(\xi)\right) \mathbf{e}_{k}, \\
& \boldsymbol{\varphi}^{h p}\left(x^{\mathbf{1}}\right)=\left(\sum_{j=1}^{\boldsymbol{m}} \varphi_{k}^{(j)} \phi^{(j)}(\xi)\right) \mathbf{e}_{k}, \\
& \boldsymbol{\Psi}^{h p}\left(x^{\mathbf{1}}\right)=\left(\sum_{j=\mathbf{1}}^{\boldsymbol{m}} \psi_{k}^{(j)} \phi^{(j)}(\xi)\right) \mathbf{e}_{k}, \\
& \boldsymbol{\theta}^{h p}\left(x^{\mathbf{1}}\right)=\left(\sum_{j=\mathbf{1}}^{\boldsymbol{m}} \theta_{3}^{(j)} \phi^{(j)}(\xi)\right) \mathbf{e}_{3}, \\
& k=1,2 .
\end{aligned}
$$

The adopted basis functions $\phi^{(j)}$ are $C^{0}$ interpolant polynomials of Gauss-Lobatto-Legendre (GLL) quadrature points [26], which are suitable for high-order expansions. Explicitly, the one-dimensional basis functions of the order $p=m-1$ are expressed using the $p$-order Legendre polynomial $P_{m-1}$, as shown

$$
\phi^{(j)}(\xi)=\frac{\left(1-\xi^{2}\right) P_{m-1}^{\prime}(\xi)}{m(m-1) P_{m-1}\left(\xi_{j}\right)\left(\xi-\xi_{j}\right)}
$$

\section{NUMERICAL RESULTS}

In the following section cantilever and simply supported microbeams are evaluated using a 4-element mesh with $P=4$ interpolation functions for primary variables to avoid shear locking. Nondimensional parameters are used according with the following equations:

$$
\begin{gathered}
\hat{\mu}=\mu / E, \quad \hat{\kappa}=\kappa / E, \\
\hat{\beta}_{2}=\beta_{2} /\left(E L^{2}\right), \\
\hat{\beta}_{3}=\beta_{3} /\left(E L^{2}\right), \\
\hat{I}_{2}=I_{2} /\left(A L^{2}\right), \\
\hat{R}_{2}=L / \sqrt{\left(I_{2} A\right)} \\
\hat{f}_{2}=f_{2} /(E A), \\
\hat{m}_{3}=m_{3} /(E A), \\
\widehat{U}_{2}=3 E I_{2} u_{2}\left(x^{1}\right) /\left(f_{2} L^{3}\right),
\end{gathered}
$$

where $I_{2}$ is the moment of inertia, $R_{2}$ is the slenderness ratio, $f_{2}$ the point load at the free end of the beam, $A$ the area of the cross-section and $L$ the length of the beam

\section{A. Convergence analysis}

Preliminary converge analysis was conducted to demonstrate the numerical stability of the proposed finite element model applied to micropolar elasticity. A cantilever beam was analyzed with the nondimensional parameters in Table I [17].

TABLE I

\begin{tabular}{|c|c|}
\hline Parameter & Value \\
\hline$\hat{R}_{2}$ & 10 \\
\hline$\hat{\mu}$ & $3 / 8$ \\
\hline$\hat{\kappa}$ & 0.02 \\
\hline$\hat{\beta}_{2}$ & 0 \\
\hline$\hat{\beta}_{3}$ & $5 \times 10^{-4}$ \\
\hline
\end{tabular}

Vertical deflection at the free end of the beam was calculated for different $P$ levels. A normalized vertical deflection $\underline{u}_{2}$ is compared for different numbers of nodes considering the case with $P=8$ and 8 elements as the reference value, being $\underline{u}_{2}$ calculated by the following equation 


$$
\underline{u}_{2}=\frac{u_{2}(P, N)}{u_{2}(8,8)} .
$$

The cantilever beam is loaded with a nondimensional point load at its free end $\hat{f}_{2}=5 \times 10^{-6}$ and a distributed body moment $\widehat{m}_{3}=10^{-6}$. Fig. 1 shows the convergence analysis. An excellent rate of convergence is achieved when higherorder elements are employed.

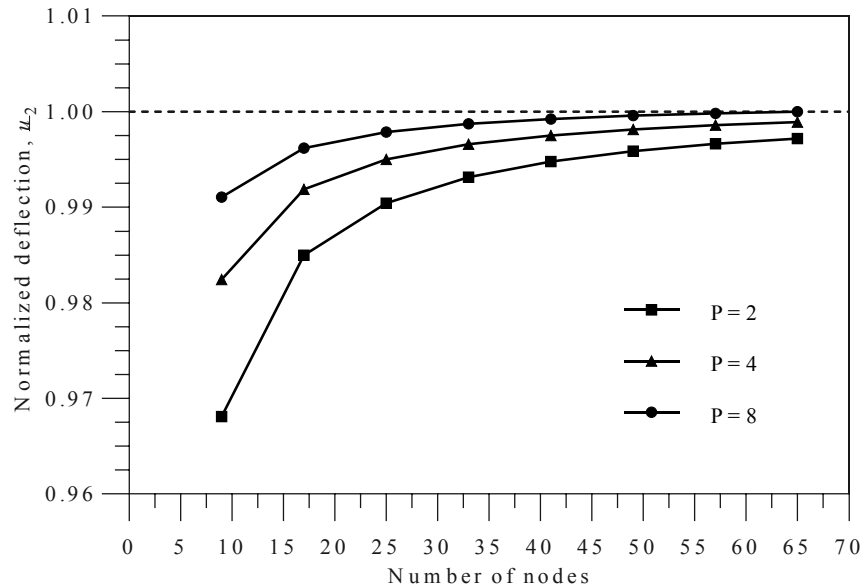

Fig. 1 Convergence analysis.

\section{B. Bending analysis of micropolar beams}

For the evaluation of the proposed model, results are compared against [17]. In the first case, a cantilever beam with a squared cross-section is considered. The action of different distributed body moments $\widehat{m}_{3}=0, \pm 0.5 \times 10^{-6}, \pm 1 \times 10^{-6}$ and a point load applied at the free end $\hat{f}_{2}=5 \times 10^{-6}$ are analysed. The material parameters used in the results are given in Table I. The boundary conditions are taken as follows:

$$
\begin{gathered}
u_{1}(0)=u_{2}(0)=0, \\
\varphi_{1}(0)=\varphi_{2}(0)=0, \\
\psi_{2}(0)=0 .
\end{gathered}
$$

Fig. 2 shows the normalized displacement $\widehat{U}_{2}$ along the length of the beam. The advantage of the present formulation is that $3 \mathrm{D}$ constitutive relations are used. Hence, the inclusion of the Poisson ratio leads to more stiff results. For the case with a distributed moment of $\widehat{m}_{3}=+1 \times 10^{-6}$, the proposed model presents displacements $10.86 \%$ smaller in comparison with the reference value. The same behaviour is evidenced in fig. 2 and fig. 3 with respect to macrorotation $\varphi_{1}$ and microrotation $\theta_{3}$ respectively.

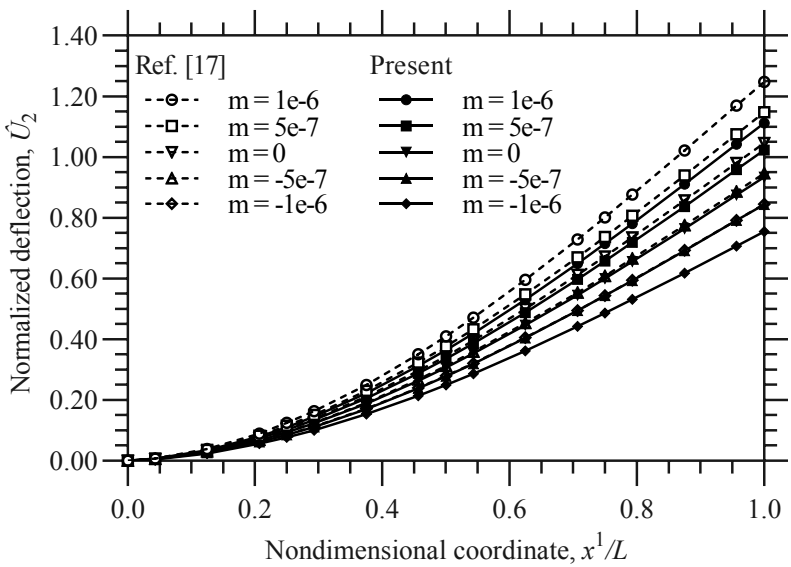

Fig 2. Normalized vertical deflection $\hat{U}_{2}$ of the cantilever beam.

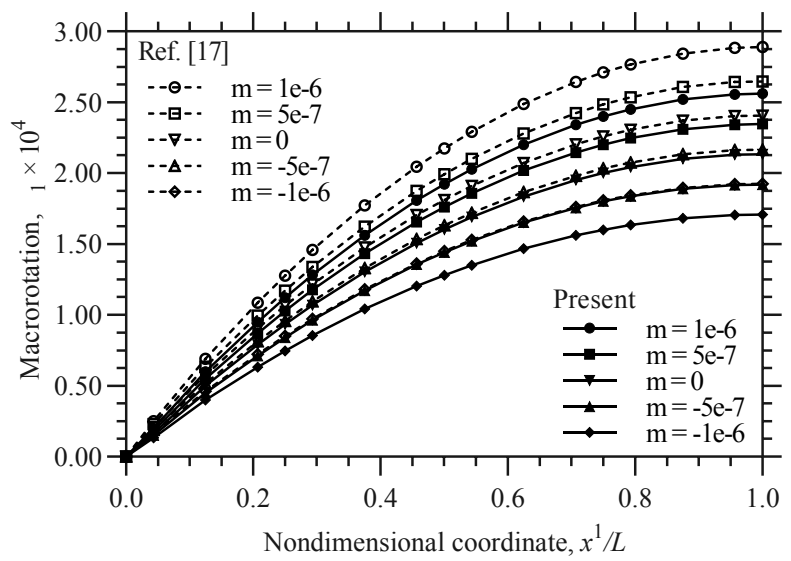

Fig 3. Macrorotation $\varphi_{1}$ of the cantilever beam.

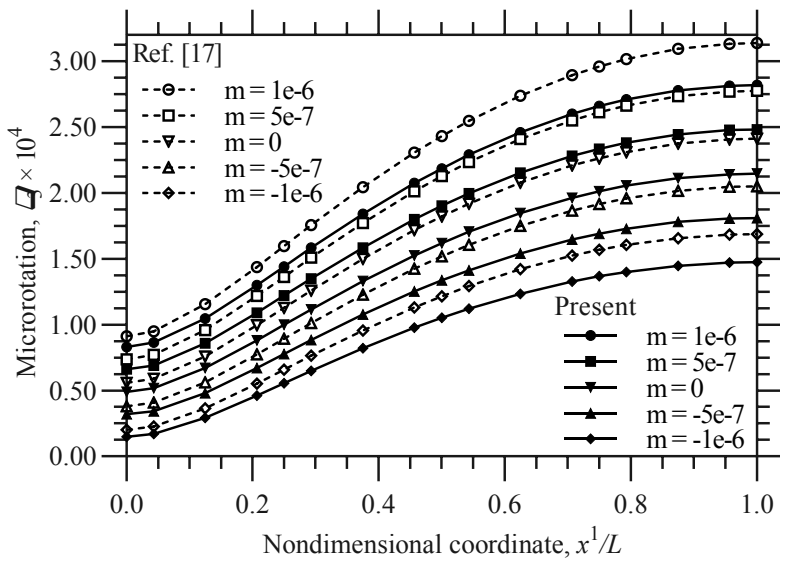

Fig 4. Microrotation $\theta_{3}$ of the cantilever beam.

Secondly, a simply supported micropolar beam is evaluated under the action of a distributed load $p_{2}$. The magnitude of $p_{2}$ is selected such that it generates a nondimensional vertical deflection of 0.3 at the middle spam of the beam based on the Timoshenko bending theory. The material parameters are given in Table II. 


\begin{tabular}{|c|c|}
\multicolumn{2}{c}{ TABLE II } \\
$\begin{array}{c}\text { Parameter } \\
\hat{R}_{2}\end{array}$ & Value \\
\hline$\hat{\mu}$ & 30 \\
\hline$\hat{\kappa}$ & $\left(2 \times 10^{2}, 2 \times 10^{-10}\right)$ \\
\hline$\hat{\beta}_{2}$ & 0 \\
\hline$\hat{\beta}_{3}$ & $2 \times 10^{-10}$ \\
\hline
\end{tabular}

The boundary conditions are taken as follows:

$$
\begin{aligned}
& u_{1}(0)=u_{2}(0)=0, \\
& u_{1}(L)=u_{2}(L)=0,
\end{aligned}
$$

The proposed model presents stiffer behavior compared with [17]. Fig. 5 shows the results considering the first value of micropolar constant $\hat{\kappa}$ presented in Table II. It is observed that the macrorotation $\varphi_{1}$ and microrotation $\theta_{3}$ coincide for the present formulation as it was stated by [17]. Fig. 6 shows the results with the second value of micropolar constant $\hat{\kappa}$ from Table II. It is observed that as the micropolar constant $\hat{\kappa}$ is reduced, the microrotation $\theta_{3}$ decreases in comparison with the macrorotation $\varphi_{1}$. Also, the microrotation $\theta_{3}$ obtained with the proposed model coincides with the results from [17].



Fig. 5 Bending deformations of a simply supported beam under a distributed load $p_{2}\left(\widehat{\kappa}=2 \times 10^{2}\right)$

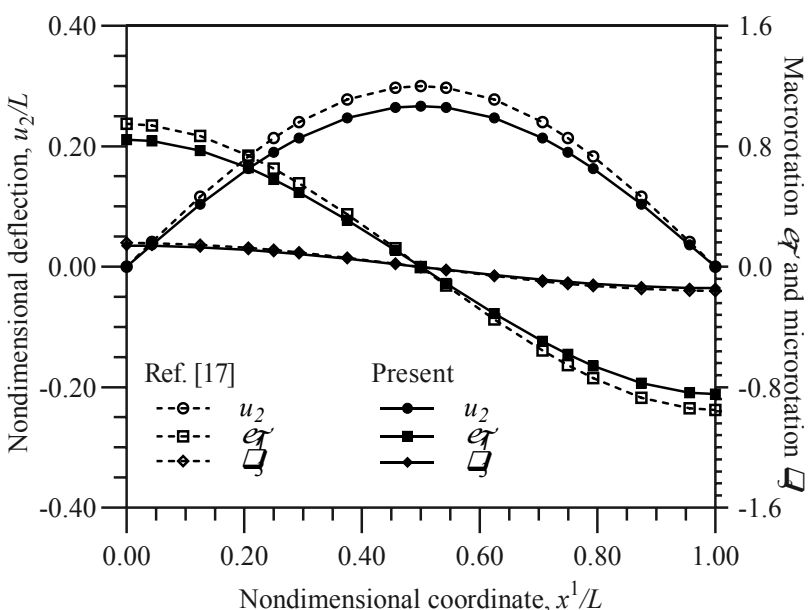

Fig. 6 Bending deformations of a simply supported beam under a distributed load $p_{2}\left(\widehat{\kappa}=2 \times 10^{-10}\right)$.

\section{CONCLUSIONS}

In the present study, a finite element model based on an improved first-order shear deformation theory was obtained based on micropolar elasticity theory. The proposed model uses six independent variables for the approximation of the displacement and microrotation fields. A variational formulation has been derived using 3D constitutive relations and spectral high-order interpolation functions. A convergence study of the vertical deflection of a cantilever beam was evaluated, showing the advantages of these spectral high-order interpolation functions. Bending results were obtained for cantilever and simply supported beams with distributed vertical loads and body moments, which were compared against the deflections of a micropolar first-order shear deformation model. The proposed model developed stiffer deflections, macro rotations, and microrotations due to the use of $3 \mathrm{D}$ constitutive equations. In the next investigations, the authors expect to extend the present research to evaluate geometrical nonlinear behavior and functionally graded materials considering micropolar elasticity theory.

\section{REFERENCES}

[1] Z. Rueger and R. S. Lakes, "Cosserat elasticity of negative Poisson's ratio foam: Experiment," Smart Mater. Struct., vol. 25, no. 5, 2016, doi: 10.1088/0964-1726/25/5/054004.

N. Shafiei, S. S. Mirjavadi, B. MohaselAfshari, S. Rabby, and M. Kazemi, "Vibration of two-dimensional imperfect functionally graded (2D-FG) porous nano-/micro-beams," Comput. Methods Appl. Mech. Eng., vol. 322, pp. 615-632, 2017, doi: 10.1016/j.cma.2017.05.007.

[3] S. Shaw, "Bending of a Thin Rectangular Isotropic Micropolar Plate," Int. J. Comput. Methods Eng. Sci. Mech., vol. 20, no. 1, pp. 64-71, 2019, doi: 10.1080/15502287.2019.1568616.

[4] S. Cuenot, C. Frétigny, S. Demoustier-Champagne, and B. Nysten, "Surface tension effect on the mechanical properties of nanomaterials measured by atomic force microscopy," Phys. Rev. B - Condens. Matter Mater. Phys., vol. 69, no. 16, pp. 1-5, 2004, doi: 
[5] D. C. C. Lam, F. Yang, A. C. M. Chong, J. Wang, and P. Tong, "Experiments and theory in strain gradient elasticity," J. Mech. Phys. Solids, vol. 51, no. 8, pp. 1477-1508, 2003, doi: 10.1016/S0022-5096(03)00053-X.

[6] A. W. Mcfarland and J. S. Colton, "Role of material microstructure in plate stiffness with relevance to microcantilever sensors," $J$. Micromechanics Microengineering, vol. 15, no. 5, pp. 1060-1067, 2005, doi: 10.1088/0960-1317/15/5/024.

[7] A. R. Srinivasa and J. N. Reddy, "An overview of theories of continuum mechanics with nonlocal elastic response and a general framework for conservative and dissipative systems," Appl. Mech. Rev., vol. 69, no. 3, 2017, doi: 10.1115/1.4036723.

[8] W. Voigt, "Theoritiscke Studien über die Elastizitätsvertältnisse der Krystalle," Abhandlungen der Königlichen Gesellschaft der Wissenschaften Göttingen, vol. 34, pp. 3-51, 1887.

[9] F. Cosserat, E., Cosserat, Théorie des corps déformables. Paris: A. Hermann et fils, 1909.

[10] A. C. Eringen and E. S. Suhubi, "Nonlinear theory of simple microelastic solids-I," Int. J. Eng. Sci., vol. 2, no. 2, pp. 189-203, 1964, doi: 10.1016/0020-7225(64)90004-7.

[11] E. S. Suhubl and A. C. Eringen, "Nonlinear theory of micro-elastic solids-II," Int. J. Eng. Sci., vol. 2, no. 4, pp. 389-404, 1964, doi: 10.1016/0020-7225(64)90017-5.

[12] A. C. Eringen, "Linear Theory of Micropolar Elasticity," J. Math. Mech., vol. 15, no. 5, pp. 909-923, 1966.

[13] A. C. Eringen, Microcontinuum Field Theories I: foundations and solids. Berlín: Springern, 1999.

[14] P. Nampally, A. T. Karttunen, and J. N. Reddy, "Nonlinear finite element analysis of lattice core sandwich beams," Eur. J. Mech. A/Solids, vol. 74, no. December 2018, pp. 431-439, 2019, doi: 10.1016/j.euromechsol.2018.12.006.

[15] S. Ramezani, R. Naghdabadi, and S. Sohrabpour, "Analysis of micropolar elastic beams," Eur. J. Mech. A/Solids, vol. 28, no. 2, pp. 202-208, 2009, doi: 10.1016/j.euromechsol.2008.06.006.

[16] R. A. Regueiro and Z. Duan, "Static and dynamic micropolar linear elastic beam finite element formulation, implementation, and analysis," J. Eng. Mech., vol. 141, no. 8, pp. 1-18, 2015, doi: 10.1061/(ASCE)EM.1943-7889.0000910.

[17] S. Hassanpour and G. R. Heppler, "Comprehensive and easy-to-use torsion and bending theories for micropolar beams," Int. J. Mech. Sci., vol. 114, pp. 71-87, 2016, doi: 10.1016/j.ijmecsci.2016.05.007.

[18] N. Ding, X. Xu, and Z. Zheng, "A size-dependent nonlinear microbeam model based on the micropolar elasticity theory," Acta Mech., vol. 227, no. 12, pp. 3497-3515, 2016, doi: 10.1007/s00707016-1672-8.

[19] V. V. Zozulya, "Micropolar curved rods. 2-D, high order, Timoshenko's and Euler-Bernoulli models," Curved Layer. Struct., vol. 4, no. 1, pp. 104-118, 2017, doi: 10.1515/cls-2017-0008.

[20] S. Roy Chowdhury and J. N. Reddy, "Geometrically exact micropolar Timoshenko beam and its application in modelling sandwich beams made of architected lattice core," Compos. Struct., vol. 226, p. 111228, 2019, doi: 10.1016/j.compstruct.2019.111228.

[21] R. A. Arciniega and J. N. Reddy, "Large deformation analysis of functionally graded shells," Int. J. Solids Struct., vol. 44, no. 6, pp. 2036-2052, 2007, doi: 10.1016/j.ijsolstr.2006.08.035.

[22] W. Pietraszkiewicz and V. A. Eremeyev, "On vectorially parameterized natural strain measures of the non-linear Cosserat continuum," Int. J. Solids Struct., vol. 46, no. 11-12, pp. $2477-$ 2480, 2009, doi: 10.1016/j.ijsolstr.2009.01.030.

[23] H. Eremeyev, V.A.,Lebedev, L.P., Altenbach, Foundations of Micropolar Mechanics. Springer-Verlag Berlin Heidelberg, 2013.

[24] S. C. Cowin, "An incorrect inequality in micropolar elasticity theory," Zeitschrift für Angew. Math. und Phys. ZAMP, vol. 21, no. 3, pp. 494-497, 1970, doi: 10.1007/BF01627956.

[25] W. Pietraszkiewicz and V. A. Eremeyev, "On natural strain measures of the non-linear micropolar continuum," Int. J. Solids Struct., vol. 46, no. 3-4, pp. 774-787, 2009, doi: 10.1016/j.ijsolstr.2008.09.027.

[26] S. Karniadakis, G. E.; Sherwin, Smectral/hp Element Methods for

$18^{\text {th }}$ LACCEI International Multi-Conference for Engineering, Education, and Technology: "Engineering, Integration, and Alliances for a Sustainable Development" "Hemispheric Cooperation for Competitiveness and Prosperity on a Knowledge-Based Economy", 29-31 July 2020, Buenos Aires, Argentina. 